\title{
Planificación tributaria como herramienta mitigadora de riesgos fiscales en la industria camaronera
}

Tax planning as a mitigating tool for risks fiscal in the shrimp industry

Deysy Soraya García Centeno. ${ }^{1}$, Ivonne Cecilia Narváez Zurita. ${ }^{2}$, Lesi Vanessa Giler Escandón. ${ }^{3}$, \& Juan Carlos Erazo Álvarez. ${ }^{4}$

DOI: https://doi.org/10.33262/visionariodigital.v3i2.1.588

\begin{abstract}
.
Currently, tax planning is a tool for taxable person that ensures the timely and voluntary compliance with the law of the tax obligations of the taxpayer for their generated activities. For this reason, knowing the principles that govern tax planning, the elements that integrate it and the benefits it provides are fundamental when managing the tax risks that threaten taxpayers.

In this article deploys a tax planning proposal is presented based on tax regulations, risk detection, financial situation analysis and compliance capacity. The planning is adjusted to the specific and unique characteristics of the taxpayer, in such a way that it takes advantage of the benefits that generate an increase in profits and a fair tax burden.

In order to develop a tax plan according to the reality of the taxpayer, at the first instance it was substantiated the literature on the subject was analyzed, second a

\footnotetext{
${ }^{1}$ Universidad Católica de Cuenca, Posgradista Maestría en Contabilidad y Auditoría, Cuenca, Ecuador, dsgarciac845@psg.ucacue.edu.ec

${ }^{2}$ Universidad Católica de Cuenca, Subdirección de Posgrado, Cuenca, Ecuador, inarvaez@ucacue.edu.ec

${ }^{3}$ Universidad Católica de Cuenca, Subdirección de Posgrado, Cuenca, Ecuador, lvgilere@ucacue.edu.ec

4Universidad Católica de Cuenca, Subdirección de Posgrado, Cuenca, Ecuador, jcerazo@ucacue.edu.ec
} 
diagnosis was made for the company Bioangelo S.A. which does business in the shrimp sector as a supplier of usual exporters. Afterwards, the proposed planning was analyzed, designed and raised a planning, considering tributary benefits among which has access as a taxpayer

Keywords: tax planning, liquidity, tax compliance, risks.

\section{Resumen}

Actualmente la planificación tributaria se ha convertido en una herramienta para el sujeto pasivo que desea asegurar un cumplimiento oportuno, voluntario y apegado a la ley, con base en las obligaciones tributarias por su actividad generadas; razón por la cual, conocer los principios que la rige, los elementos que la integran y los beneficios que aporta es fundamental al momento de gestionar los riesgos fiscales que amenazan a los contribuyentes.

En el presente artículo se despliega la propuesta de planificación tributaria con base en la normativa, detección de riesgos, análisis de la situación financiera y capacidad de cumplimiento del contribuyente. Siendo la planificación, ajustada a las características propias y singulares del sujeto pasivo, de tal forma que se pueda aprovechar los beneficios tributarios, para que así, estos aporten a un incremento en las ganancias y con ello al establecimiento de una carga impositiva justa.

Con el fin de desarrollar una planificación tributaria acorde a la realidad del sujeto pasivo, en primera instancia se fundamentó el tema con la literatura existente, luego se diagnosticó la gestión tributaria de la empresa Bioangelo S.A., la misma que se desempeña en el sector camaronero como productora y proveedora habitual de exportadores, posteriormente se analizó, diseñó y planteó una planificación, considerando los beneficios tributarios a los cuales tiene acceso en calidad de contribuyente.

Palabras claves: planificación tributaria, liquidez, cumplimiento tributario, riesgo tributario.

\section{Introducción.}

A través de la historia los impuestos se han convertido en un factor clave para el presupuesto del Estado, pues el aporte realizado por los sujetos pasivos por medio del pago de sus obligaciones tributarias, permite obtener los recursos necesarios para gestionar la inversión en obras de interés social, tales como educación, salud, seguridad y defensa. Para Aguirre 
(2010) los tributos deben ser innegables y no arbitrarios; su aplicación esta normada por una ley y cuenta con una serie de incentivos a los que el sector empresarial puede acceder, existiendo la posibilidad de realizar una planificación que permita visualizar los beneficios económicos a obtener con ello, no obstante la falta de planificación conlleva posibles dificultades para cumplir con estas obligaciones, problema que se evidencia en el retraso de la presentación de las declaraciones, solicitudes de facilidades de pago, declaración incompleta o errada, entre otros.

Ante este evento, se reconoce la importancia de estructurar una planificación tributaria, basada en la detección de riesgos (multas, glosas, clausuras, etc.) y errores (contables y administrativos) cometidos, estableciendo a su vez los deberes formales a cumplir, para con ello construir las estrategias de manera anticipada con el objetivo de mitigar, eliminar o transferir estos riesgos; para con la ejecución de este proceso determinar la base imponible sobre la cual se calcula el tributo a liquidar.

Es por lo antes indicado, que la planificación tributaria constituye una herramienta que busca direccionar las operaciones del contribuyente. No obstante, dentro de los procesos de una gran parte de organizaciones no se dispone una planificación tributaria que permita el pago oportuno de los haberes para con el Estado y la minimización de la carga impositiva con el aprovechamiento de los beneficios otorgados por el mismo (Gil, 2012). En este sentido, la planificación tributaria es un proceso constituido por una serie de actos o actuaciones lícitas del contribuyente, para invertir los recursos de manera óptima y con la menor carga impositiva que sea admisible (Carpio, 2012). Esta herramienta gerencial, debe estar enmarcada en la normativa vigente y ser modificada periódicamente con base a la realidad de la organización, siendo necesaria una evaluación y control posterior.

Para Tejada y Carrera (2010) la planificación tributaria, es aquel conjunto de alternativas que sujetas a la ley se pueden utilizar por el contribuyente o responsable empresarial durante uno o varios periodos contables, cuyo objetivo principal es el cálculo y pago del impuesto que debe ser entregado al estado, aplicando las normas legales vigentes dentro del país, es entonces una herramienta que permite mantener la estabilidad económica necesaria para la realización de operaciones empresariales.

Por su parte, Contreras (2016) manifiesta que la planificación fiscal consiste en ahorrar la carga tributaria a través de todo el ciclo o periodo fiscal de la persona natural o jurídica, manejando todas las deducciones y garantías que establece el código tributario o las leyes vigentes para el pago de impuestos de manera oportuna y veraz, buscando la reducción de la deuda tributaria a mediano y largo plazo, sin caer en morosidad por falta de fondos para 
cumplir con las obligaciones que establecen los organismos controladores en este caso la Administración Tributaria.

En tal contexto, la planificación constituye una actividad que asigna recursos para la consecución de objetivos, empleando los medios adecuados para la obtención de los mismos, tomando decisiones acertadas que faciliten el camino para lograr las metas trazadas en cualquier institución, por tanto, la importancia de la planificación tributaria radica en la reducción del riesgo de fracaso empresarial en cada etapa, el mismo que podría ocurrir por la falta de experiencia, baja competencia gerencial, desconocimiento del mercado, deficiente calidad de los productos o servicios, poco capital de trabajo empleado, falta de planificación operacional, problemas de subsistencia empresarial, entre otros, Navajo (2009).

Por lo antes expuesto, los objetivos de la planificación tributaria son:

1. Los cambios en el comportamiento del sujeto pasivo, reduciendo la intensidad en una determinada actividad sujeta a pago impositivo.

2. Los cambios en la forma de realizar las actividades para legalizar o minimizar la carga impositiva.

3. Prevenir el pago impositivo, es decir, predecir el pago o proyectarlo adecuadamente, para evitar la iliquidez en la organización,

4. Minimizar la incertidumbre en la base imponible y estructura impositiva futura, (Tejada y Carrera ,2010).

Para que estos objetivos se cumplan en las organizaciones, se requiere contar con elementos y principios esenciales que guíen el comportamiento de las personas encargadas de la planificación tributaria (Rivas ,2000; Rivas y Vergara, 2002).

Figura 1. Elementos y principios de la planificación tributaria
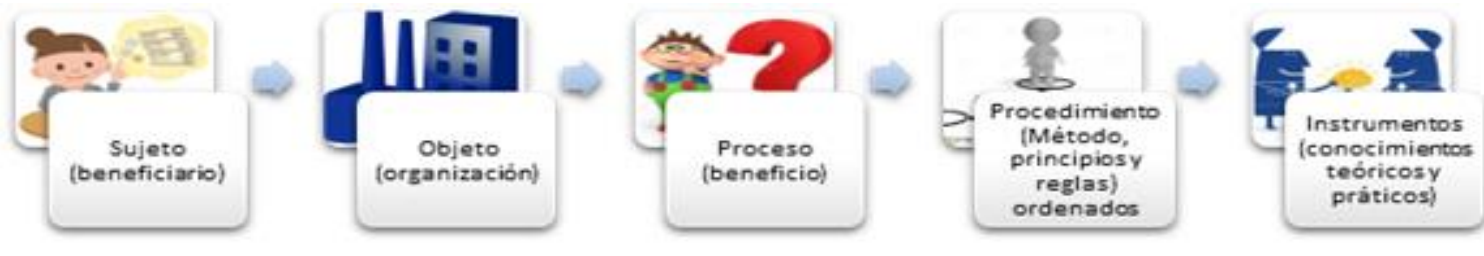

Necesidad

Legalidad

Oportunidad

Globalidad

Utilidad

Realidad 


\section{La planificación tributaria y el análisis financiero}

La planificación tributaria permite verificar la efectividad de los recursos, teniendo como aliado al análisis financiero. Hernández (2005) define al análisis financiero como una herramienta para evaluar la situación operativa de una organización, facilitando de esta manera el diagnóstico presente y la visualización de situaciones futuras, puesto que su finalidad es la obtención de objetivos establecidos con anterioridad; siendo entonces una técnica que está a disposición de la gerencia y le permite tomar decisiones estratégicas para el futuro de la organización.

Dentro de las herramientas financieras se encuentran los indicadores financieros de nivel de liquidez, razón corriente, prueba ácida, apalancamiento financiero, apalancamiento operativo, y, solvencia y rentabilidad. Brigham y Houston (2006) definen a la liquidez desde el punto de vista económico, manifestando que está dada por la disposición o dificultad de convertir un activo en efectivo disponible de manera rápida, sin que éste sufra alguna pérdida de su valor.

León (2013) asegura que la razón corriente es el indicador que verifica la disponibilidad que tiene la empresa para cubrir sus obligaciones a corto plazo, se puede decir que colabora con la gerencia en la toma de medidas futuras minimizando los riesgos, evitando que la organización sea considerada como insolvente y pierda credibilidad ante los clientes, proveedores y demás usuarios de la información empresarial.

La prueba ácida es otro índice de solvencia pero más exigente que los anteriores, González (2014) considera que éste mide el cumplimiento de sus obligaciones corrientes sin tomar en consideración su inventario, puesto que se pone de manifiesto que el inventario es un rubro que no siempre se puede vender con facilidad, teniendo como punto de partida un resultado positivo si éste se encuentra entre el 0.80 y el 1.00, si es inferior se considera que está en un punto crítico para la entidad y encasillará a la misma dentro de un grupo de empresas que no tienen capacidad de pago.

Rodríguez (2012) se refiere a la forma correcta en que una entidad económica utiliza el financiamento de las instituciones financieras, cabe mencionar que mientras más créditos financieros utilice mayor será el incremento de costos y por ende el gasto financiero, es importante también señalar que este tipo de apalancamiento hace referencia a las tasas que se pagan por estos créditos, puesto que mientras más alta sea la tasa de interés, se incrementará el gasto financiero, situación que incide en las utilidades del negocio, reduciendo las mismas. 
En cuanto al apalancamiento operativo, Oriol (1997) señala que éste constituye la superación de las ventas lo que ocasiona mayor rentabilidad y beneficios economicas de la empresa, determinándose la relacion entre los costos fijos y los costos variables, puesto que a mayor producción el incremento se realiza solo en los costos variables permitiedo a la empresa obtener utilidades mayores.

Gironella (2005) define a la rentabilidad como aquella acción que mide el rendimiento que se obtiene por la inversión de capitales en un periodo de tiempo establecido, es decir que, realiza un comparación de las utilidades obtenidas con los capitales de inversión, a este indicador además se lo conoce como rentabilidad financiera o económica, en tanto que, la solvencia constituye la capacidad que posee un ente económico para poder cumplir con sus compromisos a corto, mediano y largo plazo, es decir la diferencia entre el activo y el pasivo empresarial.

\section{Riesgo tributario.}

Actualmente, las empresas están en constante búsqueda de mecanismos que permitan la consecución de objetivos sin poner en riesgo la estabilidad operativa y económica de la misma, por lo que se dotan de herramientas que permitan un funcionamiento eficiente y un manejo adecuado de los recursos que posee, siendo necesario para ello el poder establecer estrategias que mitiguen, transfieran o eliminen los riesgos.

En general el riesgo constituye la posibilidad de que ocurra un contratiempo o evento negativo que produzca daño a la persona o entidad, pudiendo este contratiempo generarse en cualquier entorno de la vida cotidiana o actividad empresarial.

Para Pérez (2014) estos riesgos pueden clasificarse en:

$\checkmark$ Riesgo de cambio: nace de las variaciones monetarias y pueden ser por transacción.

$\checkmark$ Riego de traslación: afectan a diferentes partidas del estado financiero.

$\checkmark$ Riesgo de interés: afecta a entidades que poseen obligaciones con terceros por el cambio en el tipo de intereses.

$\checkmark$ Riesgo de valores: cuando la cartera que poseen las empresas es variable acorde al precio de los mercados de valores.

$\checkmark$ Riesgo de liquidez: cuando la institución no puede afrontar las obligaciones en el vencimiento.

La administración tributaria determina elementos o normas ante estos riesgos o fenómenos que se presentan en un momento dado, y que afectan a los contribuyentes, por ejemplo el cambio de tarifa del impuesto al valor agregado a un porcentaje mayor, el cambio en la tabla 
para el pago del impuesto a la renta, o porcentajes de retención en los bienes y servicios adquiridos o vendidos dentro de un ciclo económico (Castillo y Castillo, 2016).

Por tanto, el riesgo fiscal se considera como la discrepancia entre la norma legal promulgada por el gobierno y la forma en que la empresa la observa o interpreta, esto enmarcado en el desconocimiento de las normas tributarias internas, la falta de conciencia de la importancia de los tributos para la gestión pública y el cambio constante de las normas tributarias; siendo los factores que ponen en riesgo la estabilidad empresarial al enfrentarse a sanciones por parte de la administración tributaria debido al incumplimiento con cada una de las obligaciones fiscales a las que están sujetas las personas naturales o físicas y las organizaciones que componen el sistema económico de la nación (Pérez, 2015). Así mismo indica que, los riesgos fiscales se clasifican en:

$\checkmark$ Riesgos compliance: nacen de la falta de cumplimiento tributario.

$\checkmark$ Riesgo de análisis técnico: por la que adoptan incorrectas decisiones tributarias.

$\checkmark$ Riesgo de implementación: nace de la falta de implementación de procedimientos tributarios.

$\checkmark$ Riesgo por falta de comunicación interna.

$\checkmark$ Riesgo externo: se desprende de la falta de conocimiento de cambios en la rama tributaria.

Es importante establecer el tipo de riesgo que puede presentarse dentro del manejo contable habitual del contribuyente, debido a que este proceso es el sustento sobre el cual se presenta y desarrolla la información tributaria a ser declarada, y a su vez de donde se configura la base imponible sobre la cual se calcula el impuesto a liquidar; para de esta manera establecer los correctivos mediante estrategias que anulen o mitigen estos riesgos.

García y Paredes (2014) manifiestan que las estrategias deben ser específicas y concretas en todos los ámbitos, en especial las que tienen que ver con los recursos, acciones y personas; con el fin de definir planes que permitan la consecución de los objetivos previstos por la dirección y lograr en conjunto la realización de las metas y objetivos. De la misma manera, las estrategias de inversión son importantes, Nogales (2016) asegura que éstas permiten entender la realidad de los mercados para poder visualizar inversiones que a futuro otorgen rendimientos económicos altos, dotando de herramientas y sistemas de gestión que minimizan el riesgo y maximizan la confianza en el sistema.

La aplicación de exoneraciones, exenciones y deducciones llevan consigo un riesgo tributario implícito al ser aplicados de una manera errónea por parte del contribuyente o responsable, ya sea de forma consciente o no, debido a que éstos pueden concebir una evasión o elusión de impuesto y con ello la posibilidad de recibir sanciones por defraudar al estado; es más 
algunas sociedades confunden los términos de evasión y elusión tributaria, con planificación tributaria.

\section{Evasión y elusión tributaria.}

La evasión de impuestos es la utilización de métodos en contra de la ley para minimizar el pago de obligaciones tributarias, mediante la disminución de impuestos o la presentación de gastos irreales, comportamiento que se presenta con mucha frecuencia en las pequeñas y medianas empresas al tratar de mantenerse en el mercado y competir con las grandes empresas. En tanto que, la elusión es la disminución del pago de impuestos sin cometer delitos tributarios, sino utilizando las normas y su interpretación para el no pago de impuestos o pagar menos valor del que le correspondería (Rojas,2015).

Roca (2009) por su parte menciona que, la evasión de impuesto se da por la falta de cultura tributaria, esta situación a través de la historia ha constituido una de las razones por las cuales la administración tributaria ha tenido que adoptar medidas para la detección de este tipo de delitos fiscales; la elusión por otra parte es la acción por la cual el contribuyente intenta disminuir o desaparecer el pago de impuesto, perjudicando de esta manera al estado y el desarrollo de obras sociales.

Montaño (2007) señala que, una de las consecuencias de la evasión de impuestos es la doble imposición económica, debido a que la administración tributaria puede verificar la falta de conciencia tributaria en las recaudaciones del impuesto; situación que provoca delitos fiscales que van en contra de cada uno de los habitantes del país, al representar la no consecución de obras sociales. Este fenómeno debe ir desapareciendo acorde al cambio o incremento de los controles fiscales para contrarrestar la evasión de impuestos, permitiendo a la administración tributaria poseer mayores recursos para invertir en la sociedad.

En otras palabras, la evasión y elusión tributaria son mecanismos que pretenden la no contribución de impuestos o la disminución de los mismos, la primera busca mecanismos ilícitos para perjudicar al Estado, en tanto que la segunda, pretende mediante interpretaciones de la normativa encontrar vacíos legales para beneficiarse con la reducción de la base imponible o el no pago de impuestos, por lo que estos dos mecanismos buscan que el contribuyente no realice el pago correspondiente de impuestos, debido a la falta de cultura tributaria en el país.

\section{Caracterización del impuesto a la renta en Ecuador.}

Andino (2009) expresa que el impuesto a la renta es el eje principal de todos los sistemas tributarios, en virtud de que nace del principio de la capacidad de pago de los individuos o 
entidades jurídicas que realizan alguna actividad económica, bajo el precepto de la generación de ingresos para redistribuir equitativamente la riqueza, y crear un sistema más justo donde se trate de solventar las necesidades básicas para la subsistencia de los individuos que viven en la sociedad; sin embargo, la globalización al traer consigo la integración de economías y corporaciones en un mercado mundial, provoca dificultad para la aplicación de tributos en empresas que cuentan con modelos de negocios globales, debido a que el ente de control impone nuevas formas de evaluar el correcto cumplimiento del contribuyente, por esta razón, se deben implantar procedimientos que ayuden a la organización a cumplir con la presentación de la información tributaria acorde a la realidad y dentro de los plazos para ello establecidos (Mendoza, 2016).

La Asamblea Nacional Constituyente (2016) manifiesta que se considerarán de fuente ecuatoriana a los ingresos que obtengan las personas naturales, sucesiones indivisas y las sociedades nacionales o extranjeras, a título gratuito u oneroso, provenientes del trabajo, del capital o de la combinación de éstas en dinero, especies o servicios, además también contempla los ingresos obtenidos en el exterior por personas naturales que estén domiciliadas en el país o por sociedades nacionales que establezca la ley. Para el Servicio de Rentas Internas SRI (2016) son ingresos de fuente ecuatoriana los que perciban los ecuatorianos y extranjeros por la realización de actividades laborales, profesionales, comerciales dentro del país, salvo aquellos que sean percibidos por personas naturales que no residen en el Ecuador, y que presten servicios ocasionales dentro del mismo, siempre y cuando la permanencia de estos no supere los seis meses, este impuesto se regulariza de acuerdo a la actividad, monto a que este expuesto el contribuyente y a los beneficios o deducciones que tenga derecho.

Cabe mencionar que el contribuyente no tributa sobre el 100\% de sus ingresos, puesto que la ley faculta a los contribuyentes deducirse aquellos valores que desembolsan para realizar sus actividades económicas denominándolos gastos deducibles; constituidos por aquellos costos y gastos que puedan ser imputables al ingreso y que se sustenten en comprobantes de venta válidos.

Así mismo, en los países mediante políticas de Estado tratan de equiparar las condiciones entre los contribuyentes, otorgando beneficios que conlleven a configurar una carga tributaria justa para todos, mediante exenciones, exoneraciones y deducciones; así pues las exenciones y exoneraciones constituyen rebajas otorgadas por la ley en el pago de impuestos, radicando su diferencia en que las primeras son una dispensa legal y no necesitan consentimiento alguno para su aplicación; mientras que las segundas necesitan de la aprobación por parte del Ejecutivo mediante resoluciones. 
Al respecto Hidalgo (2017) señala que las exenciones fiscales se presentan en varias formas, siendo su finalidad el reducir o eliminar por completo la obligación a pagar del impuesto, teniendo el contribuyente derecho a una exención en la declaración de impuestos. Por otra parte, la Asamblea Nacional Constituyente (2015), estable que las deducciones son los gastos e inversiones que se efectúen con el propósito de obtener, mantener y mejorar los ingresos de fuente ecuatoriana que no estén exentos.

Montaño (2007) por su parte sostiene que están exentos los dividendos y utilidades calculados después del pago del impuesto a la renta distribuidos por sociedades nacionales o extranjeras residentes en el país, inversiones no monetarias, enajenación ocasional de inmuebles, reinversión de utilidades, ganancias de capital, entre otros; serán deducibles aquellos intereses de deudas contraídas para la actividad principal de la empresa, impuestos, tasas y contribuciones que aporten a la seguridad social obligatoria, primas de seguros, pérdidas comprobadas por casos fortuitos, fuerza mayor o dolos que afecten económicamente a la estabilidad empresarial, los sueldos y salarios a empleados, etc.

Por otra parte, dentro del aspecto tributario es preciso señalar que en Ecuador, los contribuyentes se ven obligados al cumplimiento de deberes asociados a su actividad comercial, siendo éstas: a) la obtención del RUC, b) la emisión y entrega de comprobantes de venta autorizados, c) la elaboración de libros y registros de la contabilidad, d) la presentación de las declaraciones y pago de impuestos y, e) acudir a las oficinas del SRI cuando ésta requiera su presencia (Asamblea Constituyente Nacional, 2018).

Dentro del cuarto deber formal se encuentra las obligaciones que debe cumplir el contribuyente, en el caso de las personas jurídicas o sociedades son: presentación mensual de la declaración del Impuesto al Valor Agregado (IVA), declaración de impuesto a la renta sociedades, anticipo del impuesto a la renta y retenciones en la fuente de renta, anexo de retenciones en la fuente por relación de dependencia (RDEP), anexo transaccional simplificado (ATS), declaración patrimonial (DPT) y anexos de accionistas, partícipes y socios, miembros de directorio y administradores (APS), documentos necesarios para el análisis y estructuración de la planificación tributaria.

\section{Caracterización del impuesto al valor agregado (IVA) en Ecuador}

La Asamblea Constituyente Nacional (2018) establece que el IVA es aquel valor que se grava a las transferencias de dominio de aquellos bienes importados de naturaleza corporal, en cada una de las etapas de comercialización, de igual forma a los derechos de autor, propiedad industrial, y servicios que se prestaren de acuerdo a lo que establece la ley, teniendo en consideración la definición de transferencia, que constituye el acto o contrato realizado por personas o sociedades cuya finalidad sea trasladar el dominio de bienes tangibles. 
Al igual que el impuesto a la renta, el IVA cumple un rol muy importante debido a que es el impuesto que mayores recursos genera; su estructura impositiva contempla los ingresos por ventas de bienes o servicios y los gastos asociados directamente con la actividad desarrollada. Además, considera tratamientos especiales que existen para sectores considerados estratégicos por parte del estado. Estas consideraciones se relacionan directamente con la tarifa de impuesto que gravarán los productos o servicios que se comercializan en estos sectores. Este beneficio se detalla en la tabla 1; dentro de este grupo está el sector exportador y los proveedores directos de éstos.

Tabla 1. Incentivos tributarios

\section{Reglamento de aplicación a la ley de régimen tributario interno}

Artículo 72.- IVA pagado en actividades de exportación

Artículo 180.- Devolución de IVA en la adquisición de activos fijos
Beneficio tributario para el IVA

Se le otorgará a las personas naturales y sociedades que se dediquen a producir bienes que se exporten, que hubiesen realizado adquisiciones con tarifa del IVA, en bienes, materias primas y activos fijos que se utilicen para el proceso productivo, siempre que este IVA no se haya aplicado al costo o gasto dentro de la declaración realizada.

Los sujetos pasivos de IVA, que, de acuerdo con la ley, tienen derecho a la devolución del IVA pagado en la compra local o importación de activos fijos. Para el caso de exportadores, se aplicará el factor de proporcionalidad que represente el total de exportaciones frente al total de las ventas declaradas.

\section{Fuente: Servicio de Rentas Internas}

\section{Metodología.}

El trabajo de investigación fue no experimental, en vista que se ha realizado un análisis de la información financiera, contable y tributaria de la empresa Bioangelo S.A., dedicada a la crianza de camarón, como proveedor de exportadores habituales, sin manipulación alguna de los datos. El enfoque fue mixto al haberse empleado herramientas cualitativas y cuantitativas, en la elaboración de las bases teóricas de sustento y valoración de los criterios, permitiendo identificar patrones de comportamiento en el ámbito contable, financiero y tributario. En cuanto al alcance, fue explicativo al aportar detalles sobre los componentes que se deben observar en la planificación tributaria como herramienta mitigadora de riesgos, y descriptivo en la interpretación de los resultados del diagnóstico.

Además, se utilizó los métodos: inductivo - deductivo y análitico - sintético, se aplicó varios instrumentos de investigación, entre ellos: una encuesta que fue dirigida a los administradores, accionistas, contador y auxiliar contable; mediante el diseño de un cuestionario que contenía preguntas estratégicas con base a indicadores de riesgos detectados; también se le realizó una entrevista mediante una conversación directa con el 
gerente de la empresa, basada en una guía de preguntas, que permitierón contrastar la información obtenida en las encuestas; la revisión documental, que aportó a verificar y solventar dudas, mediante la utilización de una ficha documental $\mathrm{y}$, por último; la observación directa, que permitió describir y explicar en forma clara los eventos y situaciones detectadas en los departamentos o unidades que están expuestas a errores u omisiones en el ámbito tributario.

La investigación tuvo dos universos de estudio: el primero estuvo conformado por las personas que intervinieron en el cumplimiento de las obligaciones tributarias, siendo éstos el contador y auxiliar contable; el segundo se constituyó por aquellas personas que estaban directamente relacionados con la administración financiera y operativa de la empresa, como son el presidente, socios, administrador y gerente de Bioangelo S.A.

\section{Resultados.}

Una vez que se aplicaron los instrumentos de investigación para valorar la situación tributaria de la empresa objeto de estudio, se obtuvieron los siguientes resultados:

\section{$\checkmark$ Capacidad de cumplimiento de obligaciones tributarias.}

La capacidad de pago que posee un contribuyente, tiene estrecha relación con los niveles de ingresos, costos y gastos realizados por la empresa, debido a que la primera debe contar con políticas claras de manejo de ventas al contado y crédito, e inversiones realizadas con los excedentes de dinero, mientras que las dos últimas deben estar ejecutadas con apego a lo que determina la normativa tributaria, todo esto con el fin de no generar egresos de dinero que tributariamente no reducen la base imponible.

Del diagnóstico efectuado sobre el indicador se determinó que la empresa carece de capacidad de cumplimiento de sus obligaciones tributarias de largo plazo y en algunas ocasiones de corto plazo. En lo referente a la veracidad de la información presentada, se evidencia un cumplimiento parcial, debido a que en algunos periodos donde la obligación tributaria se presenta de forma mensual, la información es subida de manera incompleta a la plataforma del Servicio de Rentas Internas, diseñado para la carga y elaboración de las declaraciones, anexos y demás obligaciones que deben ser cumplidas por este medio, para posteriormente generar una declaración sustitutiva con la información final.

\section{$\checkmark$ Estado actual de liquidez.}

La liquidez en una empresa es importante para el desarrollo del proceso productivo o de comercialización, siendo preponderante que este indicador arroje valores considerados como saludables en el ámbito de las finanzas, de no presentar esta característica es necesario buscar 
mecanismos que permitan garantizar la liquidez y por ende la permanencia de la empresa en el mercado.

En cuanto a este indicador, se estableció que Bioangelo no posee la liquidez necesaria para el desarrollo de las actividades diarias, puesto que existen pagos corrientes que han entrado en morosidad, ya que el índice de liquidez inmediata es de 0,02 (recomendable a partir de 1); situación causada por la fluctuación a la baja de los precios del producto comercializado, generando un desbalance en la cuenta de ingresos. Pudiendo este problema agravarse, ya que la empresa no verifica este indicador, y por ende no establece estrategias de mejora.

\section{$\checkmark$ Disponibilidad.}

El financiamiento a corto plazo es un factor necesario para el desarrollo de las actividades, más aún conociendo que el buen manejo de estas obligaciones genera confianza en los entes de financiación; este indicador muestra que tan eficiente es la organización para generar recursos que permita el pago oportuno de los compromisos adquiridos a corto plazo, sin descuidar el desarrollo eficiente de las operaciones diarias.

Al respecto, se detectó en Bioangelo S.A., la falta de recursos disponibles para poder hacer frente a las obligaciones tributarias de corto plazo (IVA y retenciones), puesto que debe realizar financiamiento o dejar de pagar otras obligaciones, eliminando el riesgo tributario y generando un riesgo financiero, administrativo y operacional. Cabe indicar que este tipo de obligación tributaria no debería generar estos inconvenientes al sujeto pasivo, ya que este tipo de tributos son percibidos directamente del cliente, ahora bien, otro factor a considerar es que se puede estar generando esta iliquidez por no solicitar la devolución de impuestos que no pueden ser cruzados en la declaración.

\section{$\checkmark$ Solvencia.}

La solvencia evalúa la capacidad que posee la empresa para garantizar el pago de las obligaciones financieras tanto a corto como a largo plazo, este indicador se encuentra estrechamente relacionado con la liquidez, debido a que es necesario que el activo corriente garantice las deudas a corto plazo y el activo no corriente las deudas a largo plazo, de esta manera los usuarios de la información de la empresa tendrán confianza en la solvencia de la misma.

Es así que, se determinó que la empresa no puede hacer frente al pago de sus obligaciones de manera oportuna, debiendo recurrir a endeudamiento, incrementando el problema de liquidez y, generando la posibilidad de riesgos tributarios como sanciones por la presentación tardía o incompleta de la información. También se evidenció que no se cumple de manera oportuna 
con el pago a proveedores, afectando la credibilidad de la empresa frente a los usuarios externos.

\section{$\checkmark$ Rentabilidad.}

La rentabilidad en una organización depende de la capacidad y eficiencia productiva que esta posea, para gestionar con sus fondos el pago eficiente a proveedores, así como, solventar las obligaciones financieras y tributarias; siendo trascendental poseer credibilidad interna y externamente. La rentabilidad en negocios de cría de especias acuáticas, en primera instancia depende de la capacidad de eficiencia productiva, para posteriormente gestionar la comercialización y consecuentemente la obtención de recursos monetarios para el manejo administrativo y el pago eficiente de todas las obligaciones existentes.

$\mathrm{Al}$ respecto, al efectuar el análisis de la revisión documental se pudo verificar que los recursos no están siendo aprovechados de la mejor manera, puesto que en ocasiones existe mano de obra ociosa debido a la falta de recursos para realizar las actividades diarias, lo que está ocasionando un desembolso no justificado para la empresa.

\section{$\checkmark$ Financiación del activo no corriente.}

La programación acertada del pago de los compromisos contraídos a largo plazo, es importante para la buena salud y manejo de la entidad; si bien es importante e indispensable en algunas condiciones analizar el uso del capital de terceros para adquisiciones de activos no corrientes, también es necesario estructurar una planificación acorde a la capacidad de pago de la empresa. Ahora bien, esta planificación para el caso de actividades económicas en donde los ingresos dependen en gran medida de la incidencia de factores externos llega a ser incierta, debido a la constante fluctuación de las condiciones que determinan el volumen de ingresos a recibir.

$\mathrm{Al}$ respecto, la empresa ocasionalmente necesita acceder a financiamiento de las obligaciones tributarias con la propia administración de ésta, debido a que los recursos monetarios existentes ya se encuentran comprometidos en la ejecución normal de las operaciones productivas, habiéndose verificado en la revisión documental que la empresa obtiene préstamos constantemente.

\section{$\checkmark$ Cobertura de gastos financieros.}

La cobertura de los gastos financieros dentro de las organizaciones siempre debe constar en la planificación anual diseñada, con el propósito de evitar la falta de recursos para enfrentar desembolsos que no fueron estimados al momento de contraer una obligación a corto o largo 
plazo. Cabe indicar que se pueden suscitar erogaciones de recursos por eventos fortuitos, por lo que la organización debería poseer contingentes para afrontarlos y no tener que usar recursos ya comprometidos para otras actividades u obligaciones.

Referente a este tema, se determinó que la empresa no posee una planificación para la cancelación de obligaciones a largo plazo, lo que ha generado que, en ciertas ocasiones, los intereses por préstamos deban ser cubiertos mediante una nueva obligación financiera.

\section{$\checkmark$ Impuesto a la renta.}

El impuesto a la renta constituye un deber ineludible por parte de cada uno de los contribuyentes que tienen la obligación de presentarlo, por otra parte, el pago de este impuesto se genera luego de realizar las debidas deducciones y rebajas que contempla la ley y a las cuales solamente determinados contribuyentes pueden acceder, debiendo ser satisfecho en las formas y plazos previstos para el efecto.

En este sentido Bioangelo S.A. cumple con la obligación de la presentación de la declaración en los tiempos previstos más no en las condiciones o parámetros correctos, ocasionando que el ente administrador de los tributos solicite la realización de declaraciones sustitutivas que han determinado un mayor impuesto a pagar, conllevando al pago de multas e intereses.

\section{$\checkmark$ Retenciones en la fuente del impuesto a la renta.}

La obligación que tienen las empresas al actuar como agente de retención es de vital importancia para una efectiva recaudación de este tributo por parte del Estado, así mismo, constituye un mecanismo para aliviar el impacto de pago de este impuesto en una solo cuota al diferirlo en pagos no constantes, que posteriormente serán compensados al realizar la declaración del impuesto. Es así que, se verificó el cumplimiento de este requerimiento, aunque no dentro de los plazos establecidos por la administración tributaria, lo que ha ocasionado el pago de intereses y multas por la tenencia de recursos del estado.

\section{$\checkmark$ IVA y retenciones del IVA.}

Por su parte el IVA lo deben satisfacer todos los contribuyentes que realicen actividades económicas al transferir la propiedad de un bien o brindar servicios de cualquier índole. Este impuesto tiene mayor importancia para el estado al ser el que mayores recursos genera, siendo su control más minucioso y tecnificado en estos últimos años.

Bioangelo, al respecto presenta las declaraciones de IVA con sus debidas retenciones de manera oportuna, cumpliendo con los plazos establecidos, pero como sucede con la declaración de renta los datos ingresados son incorrectos, llevando al área encargada del 
proceso a realizar varias sustitutivas dentro del año contable, con las respectivas multas e intereses.

\section{$\checkmark$ Anexos.}

Los anexos para la administración tributaria constituyen una fuente de información sobre la cual se realizan los cruces y verificación de los datos presentados en las declaraciones de IVA por los distintos sujetos relacionados entre sí. Es decir, se determina la correcta presentación de los datos consignados en la declaración de impuestos por parte de los contribuyentes, además, también sirve como fuente de sustento para la elaboración de las declaraciones por parte de los proveedores de los obligados a la presentación de estos anexos. La oportuna presentación de éstos demuestra la transparencia de las operaciones realizadas y el correcto manejo contable y financiero que la entidad maneja.

Con referencia a este tema, la empresa presenta los anexos de manera oportuna.

\section{$\checkmark$ Exenciones y exoneraciones.}

Las exenciones y exoneraciones constituyen un mecanismo de apoyo por parte del Estado a determinadas actividades productivas o industriales que ejecutan los sujetos pasivos, permitiendo al entorno empresarial mejorar su liquidez e incrementar su nivel de productividad, mediante la reinversión de esos recursos no erogados para el pago de tributos.

A través de la revisión documental y encuestas realizadas, se determinó que la empresa nunca ha hecho uso de estas exenciones y exoneraciones, debido al desconocimiento de la normativa, desaprovechando beneficios tributarios como la devolución del IVA por ser proveedor directo de exportadores.

\section{$\checkmark$ Planificación tributaria.}

La planificación tributaria tiene como objetivo el pago justo y eficiente de las obligaciones tributarias que tiene el sujeto pasivo, dentro de la actividad que desarrolla, mediante la aplicación de exoneraciones, deducciones y demás beneficios que la ley permita para el efecto. A su vez al gestionar un eficiente manejo contable y tributario, se logra eliminar o minimizar los riesgos y efectos que pudieren generarse por la mala aplicación de la norma.

En este aspecto, se evidencia la débil cultura tributaria existente en la empresa Bioangelo, puesto que no cuenta con esta herramienta, buscando actualmente gestionar beneficios de forma aislada, sin un objetivo claro, siendo prioritario establecer los parámetros y características a observar para el diseño de una planificación tributaria acorde a las particularidades de la empresa. 


\section{Propuesta.}

La planificación tributaria (figura 2) para Bioangelo S.A., tiene como objetivo ser la herramienta que aporte al cumplimiento de las obligaciones por parte del sujeto pasivo, así como a la mitigación de riesgos y al aprovechamiento de los beneficios que otorga la administración tributaria, para con ello, generar la posibilidad de disminuir la carga fiscal y finalmente lograr la maximización de utilidades en el periodo económico; todo esto, sin que la empresa incurra en evasiones tributarias que le puedan causar perjuicios económicos.

Figura 2. Propuesta de planificación tributaria.

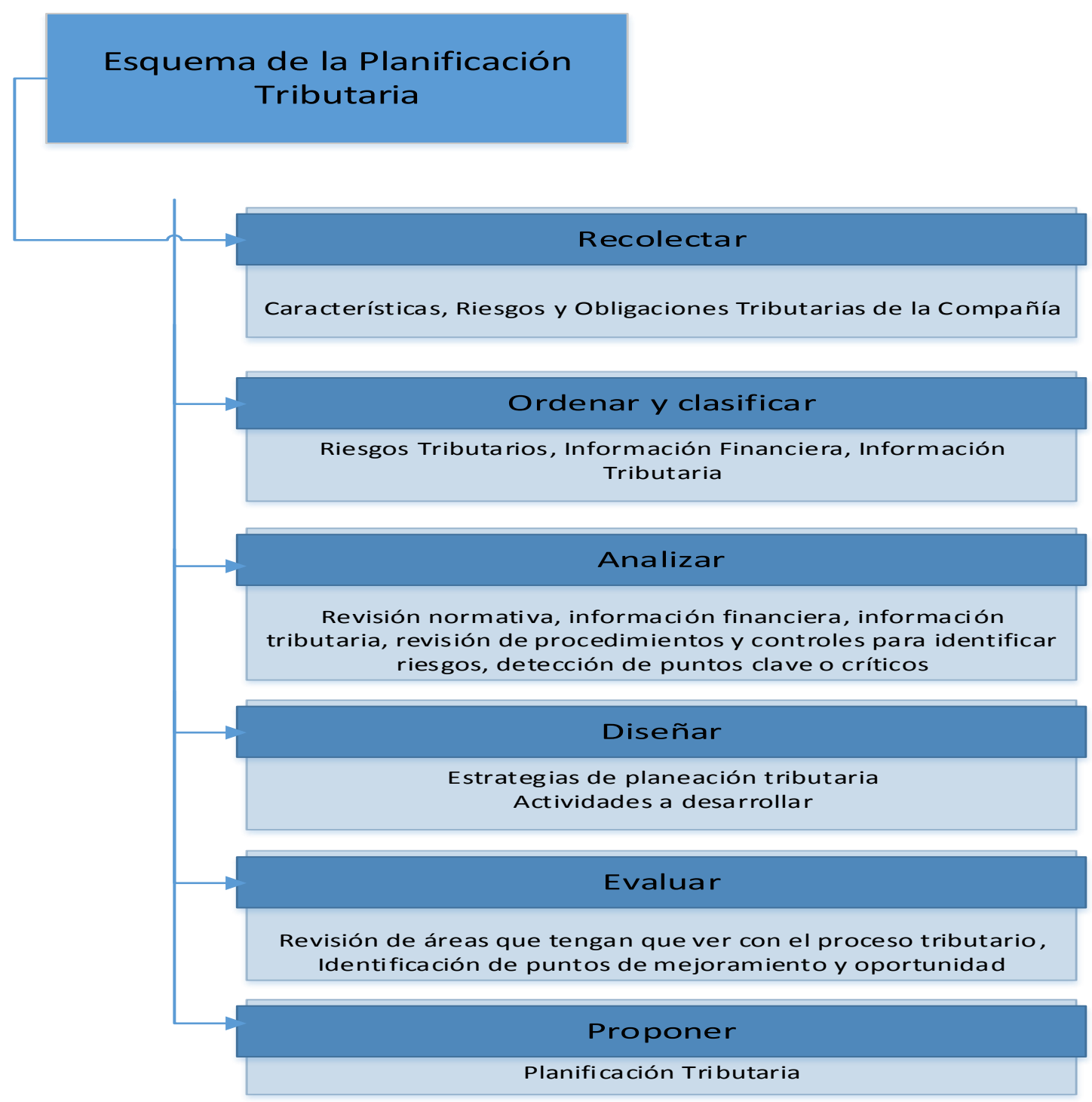




\section{$\checkmark$ Recolectar: características, riesgos y obligaciones tributarias.}

Los riesgos estratégicos del negocio están asociados a las leyes vigentes, por tanto, siendo esto un factor externo, para contrarrestarlo solamente debe ser acatado por la organización. Bioangelo deberá cumplir con cada una de las obligaciones tributarias, caso contrario se verá afectada por multas y sanciones impuestas por el SRI, lo que podría conllevar a la elaboración de constantes sustitutivas en las declaraciones, y sobre todo a pérdidas considerables en la empresa.

La actividad desarrollada por la empresa conlleva un riesgo muy alto en cuanto a resultados financieros, puesto que solo al final de la producción se puede evidenciar si se obtuvo ganancia o pérdida, la crianza del camarón implica riesgos muy altos, debido a la inversión de manera anticipada, con la venta de la producción los accionistas de la empresa recuperan la inversión de cuatro o cinco meses de trabajo, pero existe la posibilidad que en ocasiones debido a la mala administración, plagas que vienen en la larva, aplicación de productos de mala calidad, se obtengan resultados no satisfactorios, debiendo tomar decisiones inesperadas por parte de la administración de la empresa.

Bioangelo por ser una compañía es considerada un agente de retención, situación que la obliga a realizar retenciones en la fuente del IVA en todas las compras que realice, debiendo subir esta declaración y realizar el pago de la misma de manera mensual, el 100\% de sus ventas son con tarifa $0 \%$, no siendo lo mismo en la compras donde existen compras de bienes y servicios adquiridos con tarifa $12 \%$, según la información revisada de cada uno de los meses del ejercicio fiscal del año 2016, 2017 y 2018.

La presentación y pago del impuesto a la renta, se lo debe efectuar en el mes de abril, en este aspecto, a través de la revisión de información de los años 2016, 2017 y 2018 se pudo verificar las fechas de declaración, multas, intereses y contravenciones que la empresa debió enfrentar para el cumplimiento de esta obligación fiscal. Bioangelo está obligada a la presentación y pago mensual de las retenciones de Impuesto a la renta que realice por las adquisiciones efectuadas, así mismo, en este aspecto, se cuenta con información de los 12 meses de los años 2016, 2017 y 2018, que permitieron realizar la comparación con las compras efectuadas en los períodos antes descritos, verificando la correcta retención en cada una de ellas.

Las áreas tomadas en cuenta en la planificación tributaria, fueron el departamento de compras, donde se adquieren los insumos, materiales, y servicios necesarios para el correcto desenvolvimiento de las actividades de la empresa, es aquí donde se realiza el primer control de los documentos que sustentan la adquisición de los bienes y servicios, y del traspaso de 
éstos al departamento contable de manera oportuna para la realización de las retenciones respectivas.

\section{$\checkmark$ Ordenar y clasificar: riesgos tributarios, información financiera, información} tributaria.

Una vez realizada la recolección, se procedió a ordenar y clasificar esta información, el riesgo tributario estuvo vinculado a las obligaciones que debió enfrentar Bioangelo por la actividad económica que desarrolla, ya que, si la empresa no cumple con la normativa legal vigente, el riesgo de sanciones será mayor, pudiéndose enfrentar a multas o intereses que disminuirán la ganancia empresarial e inclusive a sanciones que ocasionen hasta el cierre del negocio, lo que involucra pérdidas en las actividades y disminución de las ganancias operativas, además de éstos se debe mencionar el riesgo por la falta de revisión de la documentación fuente de las operaciones realizadas por la sociedad.

Se analizó la información financiera en ingresos (ventas) y gastos (compras) operativos, al igual que los gastos no operativos realizados; las planillas de sueldos mensuales, de décimos, y fondos de reserva, para lo cual se ordenó y clasificó las compras efectuadas, con su respectivo sustento legal, asiento contable y forma de pago, junto con las declaraciones mensuales del IVA, retenciones en la fuente realizadas y recibidas, y la declaración de renta de los años 2017 y 2018. A su vez como respaldo de lo analizado se revisó la ley y reglamento de régimen tributario interno, resolución NAC-DGERCG17-00000568, reglamento de Comprobantes de Venta y de Retención, entre otros.

\section{$\checkmark$ Analizar: revisión normativa, información financiera y tributaria, revisión de procedimientos y controles, identificación de puntos clave.}

Luego de verificar el cumplimiento en la declaración y pago de impuestos para identificar riesgos de sanciones o mayores impuestos a liquidar, se detectó que no se considera como gasto financiero los intereses generados en los préstamos que realiza la empresa a instituciones financieras que ascienden a un valor de $\$ 4510,12$ para el 2017 y $\$ 5890,93$ para el 2018, eliminando con ello la posibilidad de obtener una mayor deducción y por ende una menor carga impositiva en los períodos económicos analizados.

Por otra parte, al analizar de la nómina de empleados, se evidenció que la empresa no hace uso del beneficio que otorga la administración tributaria por cada empleado nuevo ingresado, beneficio que consiste en otorgar a los sujetos pasivos del impuesto a la renta la deducción adicional del 100\% por incremento neto de empleos realizada por contratación directa en el ejercicio económico, tomando en consideración algunos aspectos indicados en la ley. 
A su vez, se debe garantizar que cada una de las adquisiciones de bienes y servicios sean sustentados con documentos legalmente autorizados por la administración tributaria, permitiendo la deducción de la carga impositiva en cada período económico. A sí mismo, dentro de la declaración de impuesto a la Renta se considera las provisiones pensiones jubilares y otras deducciones por promociones y publicidad o por contar con empleados con algún tipo de discapacidad o que tengan a su cargo dependientes con discapacidad, entre otras.

Además se detectaron falencias en la información presentada en las declaraciones del impuesto a la renta, con fundamento en las sustitutivas que se presentan en forma repetitiva a petición de la administración tributaria y que han generado más impuesto a pagar; errores causados por tener respaldos de gastos en comprobantes de venta mal emitidos; trayendo consigo el riesgo de generar obligaciones financieras para cubrir el ajuste al pago de los impuestos no considerados, riesgo que causa una reducción de la liquidez de la empresa y la eliminación de nuevas inversiones para el mejoramiento del proceso productivo.

\section{$\checkmark$ Diseñar: estrategias de planeación tributaria, actividades a desarrollar.}

Para establecer la planificación tributaria adecuada al sujeto pasivo, es imprescindible conocer el proceso productivo de la organización e identificar su posición dentro de la cadena de comercialización, información detallada en la figura 3 y 4 respectivamente, con el fin de conectar las etapas y las estrategias que pueden ser implementadas.

Figura 3. Proceso de producción camaronero.

\begin{tabular}{|ll} 
Preparacion \\
de Piscinas
\end{tabular} Llenado $\begin{gathered}\text { Siembra de } \\
\text { Larvas } \\
\text { Carvanza de }\end{gathered}$ Cosecha $\begin{gathered}\text { Venta a } \\
\text { exportador }\end{gathered}$


Figura 4. Actores involucrados en la industria camaronera.

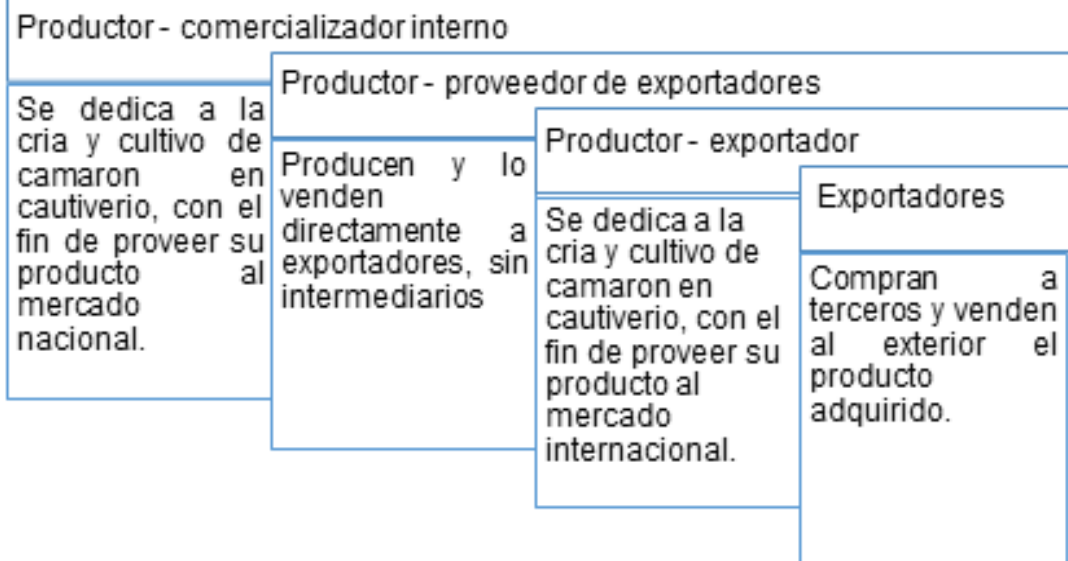

Con fundamento en lo expuesto en los párrafos que anteceden, se identifica que Bioangelo S.A., dentro de la cadena de comercialización se desarrolla como productor - proveedor directo de exportadores, llevando a cabo todo el proceso productivo desde la preparación de las piscinas hasta la cosecha, para finalmente sin intermediarios proceder a la entrega del producto al exportador. Con esta base se plantean estrategias, encaminadas a:

- Estructurar un diagrama del proceso productivo que debe llevar a cabo la organización, don de detalle por fases los insumos, cantidad de mano de obra (calificada, especializada o específica) propia o a ser contratada, materiales y maquinaria necesarios, proveedores (locales, nacionales e internacionales), riesgos que se pueden presentar en cada proceso y probabilidad de ocurrencia de estos.

- Estructurar procesos de control, que verifique el cumplimiento de requisitos que debe contener los documentos que respaldan los gastos deducibles atribuidos a la actividad que desarrolla.

- Proyectar la cantidad requerida de producción y con ello el gastos necesario a incurrir, determinando los proveedores que estarán involucrados, los meses con mayor movimiento en compras y el tipo de insumo a ser adquirido, para establecer alianzas o beneficios comerciales (descuentos por volumen en compras, mayores tiempos de crédito, descuentos por pronto pago), conocer de antemano a los proveedores para evaluar el riesgo de falta de cumplimiento o retrasos en las entregas, calidad del producto, precios, descuentos y demás beneficios que se puedan adquirir.

- Establecer los vehículos que se utilizan dentro de la organización y cuentan con un peso mayor a una tonelada, para solicitar el beneficio de la rebaja del pago de matrícula bajo el concepto de exoneración por Tonelaje (aplicado a vehículos utilizados para el desarrollo de la actividad productiva declarada).

- Analizar en el mes de diciembre del ejercicio económico en curso la normativa tributaria vigente para el periodo económico del próximo año, realizado por parte del área contable, con la finalidad de conocer los beneficios que engloban a esta industria 
y cuáles de ellas se adaptan a la organización, y establecer los requerimientos a ser ejecutados, estableciendo responsables, tipo de documento, tipo de control y evaluación del cumplimiento y beneficio que estos aportan.

- Dar a conocer la matriz anteriormente listada a los responsables de cada área involucrada, para detectar falencias, errores o procesos u factores no considerados que deben ser añadidos.

- Establecer los requerimientos tributarios de las operaciones que realiza la compañía, para de esta manera conseguir que el personal contable desarrolle con rigurosidad la verificación y revisión de los documentos que soportan el giro normal del negocio evitando por ejemplo facturas emitidas por empresas u organizaciones fantasmas.

- En junio y noviembre del ejercicio fiscal en el cual se desarrolló la planificación y aplicación de los beneficios considerados, realizar la verificación del cumplimiento mediante indicadores, y aplicar los correctivos necesarios, para reducir la pérdida de beneficios no generados.

- En marzo de cada año, verificar y organizar la documentación requerida por parte de la administración tributaria, para la aplicabilidad de la obtención de beneficios tributarios.

- En el mes de diciembre del ejercicio económico en cursos supervisar y monitorear los cambios a las normas tributarias en cada una de las áreas de la empresa: el gerente realizará el seguimiento a cada una de las estrategias implantadas, permitiendo una mejor comunicación entre los diferentes departamentos.

- Elaborar cronogramas y alertas informáticas anticipadas, sobre las obligaciones tributarias a presentar, detallando los factores que mayor control debe ejercer.

\section{$\checkmark$ Evaluar: áreas vinculadas al proceso tributario.}

Bioangelo S.A., tiene como actividad comercial única la venta de camarón (en estado natural), siendo su tarifa de IVA $0 \%$, el $31 \%$ de gastos ejecutados para el desarrollo de las operaciones que gravan IVA con tarifa $12 \%$, generando el encarecimiento de la actividad y con ello el precio de comercialización del camarón, ya que este costo debe ser incluido dentro del proceso productivo y por ende asumido por el productor camaronero. El mayor porcentaje de gastos realizados por la empresa, se encuentran en el rubro de insumos para el camarón como son: balanceados, fertilizantes, melaza, y otros, constituyendo el $60,11 \%$ del total de los costos y gastos (gravados con tarifa 0\% de IVA), en tanto que el rubro de diésel representa el $30,57 \%$ (gravados con tarifa 12\% de IVA), mientras que, entre repuestos, honorarios profesionales y seguros representan el 9,32\%.

Los procedimientos y controles que se implementarán en Bioangelo S.A. estarán enmarcados en las políticas de gobiernos, políticas empresariales, actividad principal de la empresa y beneficios económicos que se pueden obtener por resoluciones que determina la Administración Tributaria. Algunos riesgos que pueden afectar a la empresa constituyen: 
sanciones económicas por el no pago oportuno de las obligaciones financieras, sanciones económicas por la no presentación y pago oportuno de las declaraciones tributarias, sanciones por inconsistencias en el contenido de las declaraciones tributarias.

Los procedimientos de planeación tributaria repercutirán de manera directa a los resultados de Bioangelo S.A., logrando obtener mejores beneficios económicos para los socios de la empresa. Con respecto a lo antes indicado, para estos años analizados el beneficio obtenido se han obtenido mediante la solicitud de la devolución del IVA por compras relacionadas con ventas a exportadores de los valores consignados en el formulario 104 de IVA, en el casillero 510 compras tarifa $12 \%$, donde para el efecto la normativa legal indica que tienen derecho a la devolución del IVA, los contribuyentes que exporten directamente o con un exportador fijo los insumos, materias primas, servicios y activos fijos empleados en la producción de bienes que se exporten, en un tiempo que no sobrepase los 90 días, mediante una nota de crédito, cheque o transferencia.

\section{$\checkmark$ Beneficios económicos de la planificación tributaria.}

El departamento contable tributario de Bioangelo S.A., conjuntamente con los asesores contratados, establecieron que no se ha realizado la gestión para solicitar la devolución de IVA, con base en que las adquisiciones y pagos con tarifa diferente a $0 \%$ ascienden a más del $30 \%$ tanto en el año 2017 como en el 2018, valores que podrían haber servido en el pago del impuesto a la renta de ambos ejercicios fiscales y con ello reducir el riesgo por falta de liquidez, considerando que la Administración Tributaria no considera todos los gastos que se presenten para la devolución. Después de establecer la participación de utilidades, se determina un impuesto a la renta por pagar para el periodo 2017 y 2018, de gestionarse la devolución de IVA se obtendrá los siguientes beneficios económicos para la empresa:

- Valores por recuperar año 2017

- Valores por recuperar año 2018

- $80 \%$ proyectada devolución 2017

- $\quad 80 \%$ proyectada devolución 2018

- Nota de Crédito para año 2019
$\$ 34.863,21$

$\$ 47.217,23$

$\$ 27.890,57$

$\$ 37.773,78$

$\$ 65.664,35$

Cabe indicar que los valores recuperados por la devolución de IVA, han ayudado a cubrir los valores a pagar de impuesto a la renta del periodo 2014 y 2015 que fueron observados por parte de la administración. A su vez, se puedo verificar que la planificación tributaria aporto a una reducción del impuesto a pagar en el año 2018, con respecto al ejercicio 2017, como se puede ver en la tabla 2. Por otra parte, ha permitido que Bioangelo S.A. ejecute una recuperación de efectivo de $\$ 65.664,35$ en el año 2019 , los valores antes indicados son proyectados, por cuanto deben pasar por un proceso de validación para su devolución. 
Tabla 2. Variación del Impuesto a la renta pagado de los años 2018 con respecto al 2017

\begin{tabular}{llll}
\hline Cuenta & $\mathbf{2 0 1 7}$ & $\mathbf{2 0 1 8}$ & Variación $\mathbf{}$ \\
\hline Ingresos & $1.446 .554,54$ & $1.430 .458,96$ & $16.095,58$ \\
Costos y Gastos & $1.186 .084,03$ & $1.298 .804,94$ & $-112.720,91$ \\
Gastos operacionales & $215.470,21$ & $174.753,08$ & $40.717,13$ \\
Total costos y gastos & $1.401 .554,24$ & $1.298 .804,94$ & $102.749,30$ \\
Utilidad & $118.187,45$ & $131.654,02$ & $-13.466,57$ \\
\hline Impuesto a la renta a pagar & $\mathbf{4 . 1 9 2 , 7 1}$ & $\mathbf{1 7 2 5 4 , 0 6}$ & $\mathbf{- 1 3 . 0 6 1 , 3 5}$ \\
\hline
\end{tabular}

\section{Conclusiones.}

$\checkmark$ La planificación tributaria permite al sector empresarial organizar el pago de impuestos, aprovechando los beneficios que la Administración Tributaria otorga a los diferentes contribuyentes que desarrollan actividades económicas en el país; para esto se debe elaborar estrategias que permitan a la empresa cumplir puntualmente sus obligaciones tributarias, sin que esto implique el sacrificio de recursos corrientes necesarios para realizar actividades propias del giro del negocio.

$\checkmark$ Los riesgos tributarios constituyen una amenaza para las organizaciones, por tanto, es imperante el conocimiento de las leyes fiscales vigentes, y la generación de conciencia respecto al cumplimiento de cada una de ellas, para que, sin dejar de cumplir con las obligaciones empresariales cotidianas, se cumpla de manera simultánea las obligaciones fiscales en forma oportuna.

$\checkmark$ Bioangelo S.A., al no contar con una planificación tributaria que le permita acceder a los beneficios otorgados por la Administración, ha incurrido en una pérdida de recursos financieros, en vista que, por no solicitar la devolución de IVA correspondiente por adquisiciones realizadas para la producción a ser comercializada a exportadores, se ha dejado de percibir en total $\$ 82080,44$ dólares, por los periodos de los años 2017 y 2018.

$\checkmark$ Con la planificación tributaria, Bioangelo podrá identificar los riesgos generados por la incorrecta aplicación de la normativa, errores en la presentación de las declaraciones, inobservancia en la verificación de requisitos que deben contener los comprobantes de venta e incluso establecer o detectar empresas a quienes se considera empresas fantasmas, evitando erogaciones de dinero por multas y sanciones.

$\checkmark$ La planificación tributaria para Bioangelo S.A., permitirá recuperar el IVA en compras y con ello cubrir el valor a pagar por impuesto principalmente en renta. Con respecto a la declaración del IVA, esta se deberá cubrir sin ningún inconveniente, debido a que la empresa actúa como agente de percepción y traslada todo lo receptado al SRI, lo que deberá observar es el correcto ingreso de gastos asociados a la actividad. 


\section{Referencias bibliográficas.}

Aguirre, R. (2010). El tributo en el Ecuador. Revista Juridica.com.br, 75.

Andino, M. (2009). Hacia un nuevo sistema de imposicion directa: El impuesto a la renta para el Ecuador un sistema distributivo. Flacso Ecuador, 105-150.

Asamblea Constituyente Nacional. (21 de 08 de 2018). Ley de Régimen Tributario Interno. Impuesto al Valor Agregado. Quito, Pichincha, Ecuador: Departamento de Normativa Tributaria.

Asamblea Nacional Constituyente. (28 de 12 de 2015). Ley Orgánica de Régimen Tributario Interno. Obtenido de file:///C:/Users/Usuario/Downloads/20151228\%20LRTI.pdf: file://C:/Users/Usuario/Downloads/20151228\%20LRTI.pdf

Asamblea Nacional Constituyente. (29 de Abril de 2016). Ley Organica de Regimen Tributario Interno (LORTI). Impuesto a laRenta. Quito, Pichincha, Ecuador: REgistro Oficial suplemento 463.

Brigham, E., y Houston, J. (2006). Fundamentos de administración financiera. México: Cengage Learming.

Carpio, R. (2012). La Planificacion Tributaria Internacional. Revista Retos, 53-68.

Castillo, S., y Castillo, P. (2016). Un acercamiento al estudio dela cultura tributaria desde la perspectiva socioeducativa. Dialnet, 149-162.

Contreras, C. (2016). Planificación Fiscal. Madrid: Universitaria Ramón Areces.

Garcia, A., y Paredes, L. (2014). Estrategias financieras empresariales. MEXICO: PATRIA.

Gil, R. (2012). Importancia de la planificación fiscal y financiera de tu empresa. FINANZAS DIGITAL, 9.

Gironella, E. (2005). El apalancamiento financiero de cómo un aumento del endeudamiento puede mejorar la rentabilidad financiera de una empresa. Revista de Contabilidad y Dirección, 71-91.

González, Z. (2014). Análisis de la Razón Financiera del ácido. Finanzas, 1-14.

Hernández, J. (2005). Análisis Financiero. Perú: Mc Graww Hill. 
Hidalgo, Y. (2017). Politca Fiscal. Cobuec, 28-45.

León, C. (2013). Análisis e interpretación de estados financieros. México: Copyright.

Montano, C. (2007). Manual de Derecho Tributario Internacional. Quito: Corporacion editora nacional.

Navajo, P. (2009). Planificacion Estrategica en organizaciones no lucrativas. Madrid: Narcea S A.

Nogales, I. (2016). Estrategias de inversión. Espana: RAMA.

Oriol, A. (1997). Análisis económico financiero. España: Gestión 2000.

Pérez, C. (2015). Contabilidad Fiscal (Vol. Primera). México, México: Tax editores.

Pérez, E. (2014). Instrumentos derivados como instrumentos para la elusión fiscal. Revistra tributaria, 43-64. Obtenido de file:///C:/Users/Usuario/Downloads/2731Texto\%20de1\%20art\%C3\%ADculo-9140-1-10-20110314.pdf

Rivas , N. (2000). Planificacion Tributaria. Santiago de Chile: Magril Limitada.

Rivas, N., y Vergara, S. (2002). Planificación tributaria. Chile: Magril.

Roca, J. (2009). Tributacion directa en Ecuador. Evasion, equidad y desafios de diseno. Santiago de Chile: Cepal.

Rodríguez, L. (2012). Análisis de estados financieros, un enfoque en la toma de decisiones. México: Mc Grawhill.

Rojas, M. (2015). Evasion e elusion de impuestos. Bogota: norma.

Servicio de Rentas Internas. (29 de Julio de 2016). Ingresos publicos. Ingresos a la Renta. Quito, Pichincha, Ecuador: Registro Oficial.

Tejada, L., y Carrera, P. (2010). Planificación Tributaria. Loja: Universidad Técnica Particular de Loja.

\section{Ciencia}




\section{PARA CITAR EL ARTÍCULO INDEXADO.}

García Centeno, D., Narváez Zurita, I., Giler Escandón, L., \& Erazo Álvarez, J. (2019). Planificación tributaria como herramienta mitigadora de riesgos fiscales en la industria camaronera. Visionario Digital, 3(2.1.), 550-576.

https://doi.org/10.33262/visionariodigital.v3i2.1.588

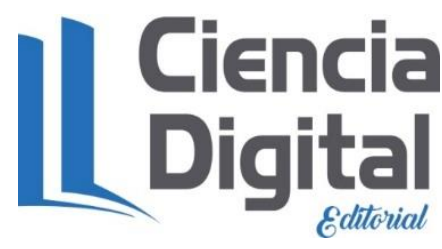

El artículo que se publica es de exclusiva responsabilidad de los autores y no necesariamente reflejan el pensamiento de la Revista Ciencia Digital.

El artículo queda en propiedad de la revista y, por tanto, su publicación parcial y/o total en otro medio tiene que ser autorizado por el director de la Revista Ciencia Digital.
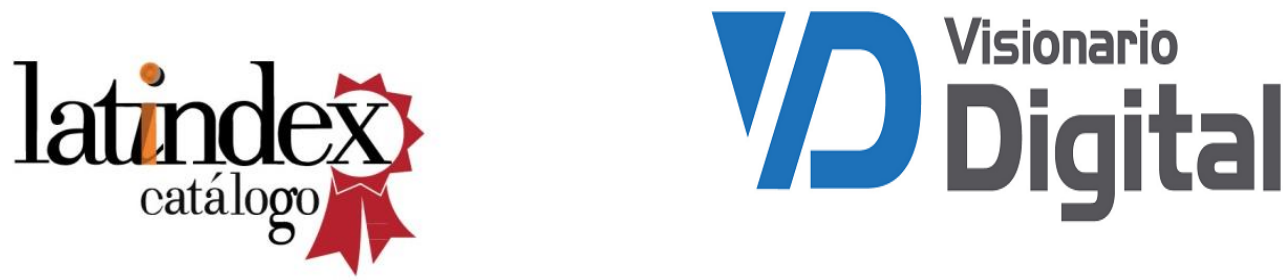